\title{
THE ALGEBRA OF LINEAR PARTIAL DIFFERENCE OPERATORS AND ITS APPLICATIONS*
}

\author{
DORON ZEILBERGER $\dagger$
}

\section{Dedicated to Richard J. Duffin}

\begin{abstract}
The algebra of linear partial difference operators is investigated, and an elimination procedure demonstrated. Applications to combinatorics are given. In particular, a new proof and a $q$-analogue of MacMahon's Master Theorem are given.
\end{abstract}

Introduction. In this paper the algebra of partial difference operators will be considered, and applications to combinatorics demonstrated. It is surprising that partial difference equations have received so little attention while partial differential equations flourished. The only serious study of partial difference equations was in numerical analysis, and then only as a tool for solving partial differential equations numerically. Specific partial difference equations arose in random walk and combinatorics, but no unified theory such as in Hörmander [6] was attempted.

We hope to show here that partial difference operators are much more comfortable to work with, since the shift operator $X f(m)=f(m+1)$ has a simple "Leibnitz rule" $X^{\alpha}(f g)=\left(X^{\alpha} f\right)\left(X^{\alpha} g\right)$. This is so much simpler than the continuous Leibnitz rule:

$$
D^{n}(f g)=\sum\left(\begin{array}{l}
n \\
k
\end{array}\right)\left(D^{k} f\right)\left(D^{n-k} g\right) \text {. }
$$

A general theory of linear partial difference equations will not be attempted here. Instead we shall. study the algebra of linear partial difference operators, and describe how to extend the elimination procedure in the algebra of polynomials (Van der Waerden [8]) to the algebra of partial difference operators. This will be followed by various applications of the elimination procedure. Unfortunately, in most cases, the algorithm is too cumbersome to be done by hand. However, since the algorithm involves nothing more complicated than multiplication by polynomials, it would be possible to employ a "symbolic" computer (such as MIT's MACSYMA) to solve some open problems in combinatorics.

This paper is dedicated to Richard J. Duffin whose pioneering work in partial difference equations prompted the author's interest in them. The author is also indebted to Richard A. Askey who challenged him to prove Andrews' [1] conjecture $(\S 5)$. The present paper is a result of attempts to prove this conjecture, which is a $q$-generalization of the already resolved Dyson's conjecture. We attempted to $q$ generalize Good's [12] elegant proof of Dyson's conjecture.

Although our algorithm is capable of doing it, in principle, for any given $n$, it turns out to be too involved to do by hand. However, our algorithm turns out to be useful in other situations, as we hope to show later.

Next, let us describe briefly the content of the paper. In $\S 1$ the algebra of linear ordinary difference operators is introduced and we show how to take an inverse of an operator. This is generalized to linear partial difference operators in $\S 2$. Following this is a description of elimination in the algebra of linear partial difference operators with

* Received by the editors January 26, 1979, and in final revised form March 24, 1980.

$\dagger$ Weizmann Institute of Science, Rehovot, Israel. This work was performed while the author was at the School of Mathematics, Georgia Institute of Technology, Atlanta, Georgia 30332. 
constant coefficients. This algorithm is then applied to give a new proof of MacMahon's [7] Master Theorem. Good's proof of Dyson's conjecture is quoted in subsection 3.4, and $\S 3$ ends with the consideration of other possible generalizations to Dyson's [8] conjecture B.

Section 4 gives a generalization of MacMahon's Master Theorem which answers, in particular, a question posed by Andrews [1, p. 213].

Andrews' [1] conjecture about the $q$-generalization of Dyson's conjecture is considered in $\S 5$. Although we failed to prove it, we describe how, in principle, for a given $n$, the algorithm is capable of settling it. Section 6 presents the algorithm of elimination in the most general setting, in the algebra of linear partial difference operators with variable coefficients.

We are very grateful to George Gasper, whose valuable criticism and comments transformed a disastrous first draft into a hopefully reasonable final version.

1. The algebra of ordinary difference operators. Let $Z$ be the set of integers. We shall here consider the vector space of functions $\mathscr{F}_{1}=\{f: Z \rightarrow \mathbb{C}\}$. A linear (ordinary) difference operator is a mapping $P: \mathscr{F}_{1} \rightarrow \mathscr{F}_{1}$ of the form

$$
P f(m)=\sum_{|\alpha|<M} c_{\alpha}(m) f(m+\alpha), \quad f \in \mathscr{F}_{1},
$$

where $M$ is a positive integer, and $c_{\alpha}$ are elements of $\mathscr{F}_{1}$. In case all the $c_{\alpha}$ 's are constants (polynomials), we have a linear difference operator with constant (polynomial) coefficients. Introducing the shift operator $X f(m)=f(m+1)$, we can write (1.1) in the form

$$
P f=\sum_{|\alpha|<M} c_{\alpha} X^{\alpha} f
$$

The set of all such operators will be denoted by $\mathscr{P}_{1}$. Note that the operator $X$ has a particularly simple "Leibnitz rule",

$$
X^{\alpha}(f g)=\left(X^{\alpha} f\right)\left(X^{\alpha} g\right)
$$

which proves that $\mathscr{P}_{1}$ is an algebra:

$$
\left(\sum c_{\alpha} X^{\alpha}\right)\left(\sum \delta_{\beta} X^{\beta}\right)=\sum_{\alpha} \sum_{\beta} c_{\alpha}\left(X^{\alpha} \delta_{\beta}\right) X^{\alpha+\beta} .
$$

Let ${ }_{+} \mathscr{P}_{1},-\mathscr{P}_{1}$ be the subalgebras

$$
\begin{aligned}
{ }_{+} \mathscr{P}_{1} & =\left\{\sum_{0 \leqq \alpha \leqq M} c_{\alpha} X^{\alpha}, \text { for some } M\right\} \\
{ }_{-} \mathscr{P}_{1} & =\left\{\sum_{-M \leqq \alpha \leqq 0} c_{\alpha} X^{\alpha}, \text { for some } M\right\} .
\end{aligned}
$$

${ }_{+} \mathscr{P}_{1}\left(\right.$ respectively $\left.-\mathscr{P}_{1}\right)$ can be embedded in the algebras of linear difference operators of infinite order:

$$
+\psi_{1}=\left\{\sum_{\alpha \geqq 0} c_{\alpha} X^{\alpha}\right\}, \quad-\psi_{1}=\left\{\sum_{\alpha \leqq 0} c_{\alpha} X^{\alpha}\right\} .
$$

The domain of an operator in ${ }_{ \pm} \psi_{1}$ is $\mathscr{F}_{1}^{0}$, the space of functions of finite (=compact) support:

$$
\mathscr{F}_{1}^{0}=\{f: Z \rightarrow \mathbb{C} ; f=0 \text { except at a finite number of points }\}
$$


An element of ${ }_{ \pm} \mathscr{P}_{1}$ has an inverse in ${ }_{ \pm} \psi_{1}$ (assume $\left.c_{0} \not \equiv 0\right)$ iff $c_{0}(m) \neq 0$ for all $m$; then

$$
\begin{aligned}
\left(\sum_{0 \leqq \alpha<M} c_{\alpha} X^{\alpha}\right)^{-1} & =\left(1+\sum_{1 \leqq \alpha<M} \frac{c_{\alpha}}{c_{0}} X^{\alpha}\right)^{-1} c_{0}^{-1} \\
& =\sum_{k=0}^{\infty}(-1)^{k}\left(\sum_{1 \leqq \alpha<M} \frac{c_{\alpha}}{c_{0}} X^{\alpha}\right)^{k} c_{0}^{-1}
\end{aligned}
$$

Each term on the right-hand side is evaluated according to (1.4), and since the lowest order term in $\left(\sum\left(c_{\alpha} / c_{0}\right) X^{\alpha}\right)^{k}$ is

$$
\left(\frac{c_{1}}{c_{0}} X\right)^{k}=\left(\frac{c_{1}}{c_{0}}\right)\left(X \frac{c_{1}}{c_{0}}\right)\left(X^{2} \frac{c_{1}}{c_{0}}\right) \cdots\left(X^{k} \frac{c_{1}}{c_{0}}\right) X^{k}
$$

we see that the sum in (1.5) is well defined, since the coefficients of any $\boldsymbol{X}^{k}$ are finite sums. This is a generalization of taking the reciprocal in the algebra of formal power series, the latter corresponding to the case where the $c_{\alpha}$ 's are all constants.

The above formalism can be applied to solve a general linear difference equation

$$
\sum_{\alpha=0}^{M} c_{\alpha}(m) f(m-\alpha)=0, \quad m \geqq M,
$$

in terms of the initial values $f(0), \cdots, f(M-1)$. Of course there is a unique solution iff $c_{0}(m) \neq 0$ for all $m$, and we can write $(1.6)$ as

$$
\left(\sum_{\alpha=0}^{M} c_{\alpha} X^{-\alpha}\right) f=0 \quad \text { in } m \geqq M .
$$

Extending $f$ by 0 in $\{m<0\}$, we get

$$
\left(\sum_{\alpha=0}^{M} c_{\alpha} X^{-\alpha}\right) f=g \quad \text { in } Z
$$

where $g$ is supported in $\{0 \leqq m \leqq M-1\}$ and each of its values is a linear combination of $f(0), \cdots, f(M-1)$.

From $\left(1.6^{\prime \prime}\right)$ we get

$$
f=\left(\sum_{\alpha=0}^{M} c_{\alpha} X^{-\alpha}\right)^{-1} g
$$

which is an explicit expression for $f$, in spite of its "formal" appearance.

2. The algebra of partial difference operators. The discussion in $\S 1$ can easily be generalized to several discrete variables. For $n$ a positive integer, consider the vector space of functions

$$
\mathscr{F}_{n}=\left\{f: Z^{n} \rightarrow \mathbb{C}\right\}
$$

A linear partial difference operator is a mapping $P: \mathscr{F}_{n} \rightarrow \mathscr{F}_{n}$ of the form

$$
P f(m)=\sum_{|\alpha|<M} c_{\alpha}(m) f(m+\alpha), \quad f \in \mathscr{F}_{n} .
$$

Where multiindex notation is used; $m, \alpha \in Z^{n}, m=\left(m_{1}, \cdots, m_{n}\right), \alpha=\left(\alpha_{1}, \cdots, \alpha_{n}\right)$, $|\alpha|=\sum_{i=1}^{n}\left|\alpha_{i}\right|$, and $M$ is a positive integer. The coefficients $c_{\alpha}(m)$ are elements of $\mathscr{F}_{n}$. In case all the $c_{\alpha}$ 's are constants (polynomials), we have a linear partial difference equation with constant (polynomial) coefficients. We introduce the shift operators $X_{i} f\left(m_{1}, \cdots, m_{i}, \cdots, m_{n}\right)=f\left(m_{1}, \cdots, m_{i}+1, \cdots, m_{n}\right), i=1, \cdots, n ;\left(X_{i}\right.$ is the unit 
shift in the $m_{i}$ coordinate). We can write (2.1) in the form

$$
P=\sum_{|\alpha|<M} c_{\alpha} X^{\alpha}
$$

because $f(m+\alpha)=X_{1}^{\alpha_{1}} \cdots X_{n}^{\alpha_{n}} f(m)=X^{\alpha} f(m)$. The set of all linear partial difference operators on $Z^{n}$ will be denoted by $\mathscr{P}_{n}$. The operators $\left(X_{1}, \cdots, X_{n}\right)$ satisfy the simple "Leibnitz rule",

$$
X^{\alpha}(f g)=\left(X^{\alpha} f\right)\left(X^{\alpha} g\right)
$$

which shows that $\mathscr{P}_{n}$ is an algebra:

$$
\left(\sum c_{\alpha} X^{\alpha}\right)\left(\sum d_{\beta} X^{\beta}\right)=\sum_{\alpha} \sum_{\beta} c_{\alpha}\left(X^{\alpha} d_{\beta}\right) X^{\alpha+\beta}
$$

Let $\Gamma_{1}, \cdots, \Gamma_{2^{n}}$ be the $2^{n}$ orthants in $Z^{n}$; then if $\Gamma$ is such an orthant,

$$
{ }_{\Gamma} \mathscr{P}_{n}=\left\{\sum_{\alpha \in \Gamma} c_{\alpha} X^{\alpha} ; \text { only finitely many } c_{\alpha} \text { 's are nonzero }\right\}
$$

is a subalgebra of $\mathscr{P}_{n}$, since $\Gamma$ is a cone.

${ }_{\Gamma} \mathscr{P}_{n}$ can be extended to the algebras of linear partial difference operators of infinite order ${ }_{\Gamma} \psi_{n}=\left\{\sum_{\alpha \in \Gamma} c_{\alpha} X^{\alpha}\right\}$.

The domain of an operator ${ }_{\Gamma} \psi_{n}$ is $\mathscr{F}_{n}^{0}$, the space of functions of finite (=compact) support,

$$
\mathscr{F}_{n}^{0}=\left\{f: Z^{n} \rightarrow \mathbb{C} ; f=0 \text { except at a finite number of points }\right\} .
$$

As a matter of fact, if $P \in_{\Gamma} \psi_{n}$ involves all $X_{1}, \cdots, X_{n}$ (i.e., it is not of lower dimension), $P$ can be applied to functions whose support is a union of "hyperstrips", i.e., functions whose support is a subset of a set of the form $\cup_{i=1}^{n}\left\{-M_{i} \leqq m_{i} \leqq M_{i}\right\}$, where the $M_{i}$ are positive integers. The space of such functions will be denoted by $\mathscr{F}_{n}^{0}$. Of course, it may happen that the domain is even larger.

An element $P$ of ${ }_{\Gamma} \mathscr{P}_{n}$ has an inverse in ${ }_{\Gamma} \psi_{n}$ (assume $\left.c_{0} \not \equiv 0\right)$ iff $c_{0}(m) \neq 0$ for all $m$, and then

$$
\begin{aligned}
\left(\sum_{|\alpha|<M} c_{\alpha} X^{\alpha}\right)^{-1} & =\left(1+\sum_{1<|\alpha|<M} \frac{c_{\alpha}}{c_{0}} X^{\alpha}\right)^{-1} c_{0}^{-1} \\
& =\sum_{k=0}^{\infty}(-1)^{k}\left(\sum_{1 \leqq|\alpha| \leqq M} \frac{c_{\alpha}}{c_{0}} X^{\alpha}\right)^{k} c_{0}^{-1} .
\end{aligned}
$$

Each term on the right-hand side is evaluated according to (2.4); since the lowest order terms in $\left(\sum_{1 \leqq|\alpha| \leqq M}\left(c_{\alpha} / c_{0}\right) X^{\alpha}\right)^{k}$ have order $k$, it is seen that the sum in $(2.5)$ is well defined, since the coefficient of any $X^{\beta}$ takes contributions only from the first $|\beta|$ terms in (2.5), and thus consists of a finite sum. This generalizes the taking of the reciprocal in the algebra of formal power series of several variables, the latter corresponding to the case where all the $c_{\alpha}$ 's are constants.

The above formalism can be applied to solve a general linear partial difference equation in an orthant $\Gamma$,

$$
\sum_{\substack{|\alpha|<M \\ \alpha \in \Gamma}} c_{\alpha}(m) f(m-\alpha)=0 \quad \text { in }\{m ; m-\alpha \in \Gamma\},
$$

in terms of the boundary values of $f$, that is, values of $f$ near the axes. Of course (2.6) has 
a unique solution iff $c_{0}(m) \neq 0$ for all $m$, and we can write $(2.6)$ in the form

$$
\left(\sum_{|\alpha|<M} c_{\alpha} X^{-\alpha}\right) f=0 \quad \text { in }\{m ; m-\alpha \in \Gamma\} .
$$

Let us extend $f$ by zero outside $\Gamma$. Then $\left(2.6^{\prime}\right)$ can be written as

$$
\left(\sum_{|\alpha|<M} c_{\alpha} X^{-\alpha}\right) f=g \quad \text { in } Z^{n},
$$

where $g$ is supported in a neighborhood of the axes, i.e., is an element of the function space $\mathscr{F}_{n}{ }_{n}$, discussed above, and each of the values of $g$ is a linear combination of values of $f$ near the axes.

From $\left(2.6^{\prime \prime}\right)$ we get

$$
f=\left(\sum_{|\alpha|<M} c_{\alpha} X^{-\alpha}\right)^{-1} g,
$$

which is an explicit expression for $f$ in $\Gamma$, in terms of its values near the axes. Suppose $\underline{P}(z)$ is a polynomial in $n$ variables, $z_{1}, \cdots, z_{n}$; then if

$$
\underline{P}(z)^{-1}=\sum_{m \geqq 0} c(m) z^{m}, \quad\left(z^{m}=z_{1}^{m^{1}} \cdots z_{n}^{m_{n}}\right),
$$

and $\delta$ is the discrete Dirac delta function:

$$
\delta(0)=1, \quad \delta(m)=0, \quad m \neq 0,
$$

then

So

$$
c(m)=\left(\sum c(\alpha) X^{-\alpha}\right) \delta(m), \quad m \in Z_{+}^{n}
$$

$$
c=\underline{P}\left(X^{-1}\right)^{-1} \delta,
$$

and $c$ satisfies the partial difference equation $\underline{P}\left(X^{-1}\right) c=\delta$, so $c$ is a fundamental solution of the operator $\underline{P}\left(X^{-1}\right)$.

3. Elimination in the algebra of linear partial difference operators with constant coefficients.

3.1 The algebra of linear partial difference operators with constant coefficients in $n$ variables, ${ }_{c} \mathscr{P}_{n}$, is isomorphic to the algebra of polynomials $\mathbb{C}\left[z_{1}, \cdots, z_{n}\right]$, and the procedure in Van der Waerden $[8, \S \S 27,77,78]$ can be used to derive equations of lower dimensions from a system of equations.

Example. Solve the system

(i) $f(m+1, n+1)-2 f(m, n+1)+f(m+1, n)=0$,

(ii) $f(m, n)+i f(m+1, n)-f(m+1, n+1)-i f(m, n+1)=0, \quad m, n \geqq 0$.

Setting $X f(m, n)=f(m+1, n), \quad Y f(m, n)=f(m, n+1)$, we can write the above equations in shorthand as

$$
\begin{aligned}
& (X Y-2 Y+X) f=0, \\
& (I+i X-X Y-i Y) f=0
\end{aligned}
$$


Let us eliminate $Y$ from

$$
\begin{aligned}
& P_{1}(X, Y)=X Y-2 Y+X, \\
& P_{2}(X, Y)=I+i X-X Y-i Y .
\end{aligned}
$$

We would like to get an operator involving $X$ only, so we write

and we get that

$$
\begin{aligned}
& P_{1}(X, Y)=Y(X-2)+X, \\
& P_{2}(X, Y)=-Y(X+i)+(I+i X),
\end{aligned}
$$

$$
Q(X, Y)=(X+i) P_{1}(X, Y)+(X-2) P_{2}(X, Y)=-2 I+(1-i) X+(1+i) X^{2},
$$

$$
\left(-2 I+(1-i) X+(1+i) X^{2}\right) f=0 .
$$

Similarly, it also satisfies

$$
\left((i+2) Y^{2}-(i+1) Y-I\right) f=0 .
$$

(3.2a), (3.2b) immediately yield $f$, given $f(0,0), f(0,1), f(1,0), f(1,1)$.

In general, given $n$ partial difference operators with constant coefficients, on $Z^{n}$, we can use the elimination procedure to obtain $n$ ordinary difference operators $Q_{1}\left(X_{1}\right), \cdots, Q_{n}\left(X_{n}\right)$. If this is the case, we say that the $\operatorname{ring}\left(P_{1}, \cdots, P_{n}\right)$ is "a complete intersection". The elimination algorithm not only yields $n$ ordinary difference operators $Q_{1}\left(X_{1}\right), \cdots, Q_{n}\left(X_{n}\right)$ in case of "complete intersection", but also tells us when the algorithm "breaks down", whenever it is not.

3.2 The elimination procedure in Van der Waerden [8] can be applied in any polynomial ring $R\left[x_{1}, \cdots, x_{n}\right]$, where $R$ is a commutative ring (there it is assumed that $R$ is a field, but for our purpose it is enough that $R$ is a commutative ring), in particular, if $R$ is the ring of partial difference operators with constant coefficients. Let $x_{1}, \cdots, x_{n}$ be $n$ indeterminates, and suppose we have $n+1$ operators with constant coefficients,

$$
P_{j}\left(X_{1}, \cdots, X_{n} ; x_{1}, \cdots, x_{n}\right), \quad j=1, \cdots, n+1,
$$

where the dependence on $x_{1}, \cdots, x_{n}$ is polynomial. In other words, the $P_{j}$ 's are polynomials in $X_{1}, \cdots, X_{n}, x_{1}, \cdots, x_{n}$. In general it is possible to obtain, by elimination, an operator $Q\left(X_{1}, \cdots, X_{n}\right)$, independent of $x_{1}, \cdots, x_{n}$, which is in the ring generated by $P_{1}, \cdots, P_{n+1}$; i.e., there exist $Q_{1}(X, x), \cdots, Q_{n+1}(X, x)$ such that $Q_{1} P_{1}+$ $\cdots+Q_{n+1} P_{n+1}$ is independent of $x_{1}, \cdots, x_{n}$.

3.3 A new proof to MacMahon's Master Theorem. MacMahon's Master Theorem (MacMahon [7], see also Andrews [1, p. 214]) asserts that the coefficient of $x_{1}^{m_{1}} \cdots x_{n}^{m_{n}}$ in

$$
\prod_{i=1}^{n}\left(\sum_{j=1}^{n} a_{i j} x_{j}\right)^{m_{i}}
$$

is equal to the coefficient of $z_{1}^{m_{1}} \cdots z_{n}^{m_{n}}$ in the power series expansion of $\left[\operatorname{det}\left(\delta_{i j}-\right.\right.$ $\left.\left.a_{i j} z_{i}\right)\right]^{-1}$.

Setting

$$
F\left(m_{1}, \cdots, m_{n} ; x_{1}, \cdots, x_{n}\right)=\prod_{i=1}^{n}\left(\sum_{j=1}^{n} a_{i j} \frac{x_{j}}{x_{i}}\right)^{m_{i}},
$$

we are interested in $F_{0}\left(m_{1}, \cdots, m_{n}\right)=$ const. term in $F\left(m_{1}, \cdots, m_{n} ; x_{1}, \cdots, x_{n}\right)$. Now

$$
X_{i} F=\left(\sum_{j=1}^{n} a_{i j} \frac{x_{j}}{x_{i}}\right) F, \quad i=1, \cdots, n,
$$


and $F$ satisfies the $n$ partial difference equations

$$
\sum_{j=1}^{n} x_{j}\left(-a_{j}+\delta_{i j} X_{i}\right) F=0, \quad i=1, \cdots, n .
$$

These are $n$ linear homogeneous equations in $x_{1}, \cdots, x_{n}$ and (in this case Gaussian) elimination yields

$$
P(X) F=0, \quad m_{1}, \cdots, m_{n} \geqq 0,
$$

where $P(X)=\operatorname{det}\left(-a_{i j}+\delta_{i j} X_{i}\right)$. Since $P(X)$ is independent of $x_{1}, \cdots, x_{n}$, and is a linear operator, we also have

$$
P(X) F_{0}=0 .
$$

We now claim that

$$
\begin{aligned}
F_{0} & =\left[X_{1}^{-1} \cdots X_{n}^{-1} P(X)\right]^{-1} \delta \\
& =X_{1} \cdots X_{n} P(X)^{-1} \delta \\
& =\left[\operatorname{det}\left(\delta_{i j}-a_{i j} X_{i}^{-1}\right)\right]^{-1} \delta .
\end{aligned}
$$

This follows from the fact that both sides are solutions of (3.3), and the boundary values match by the inductive hypothesis. By the remarks at the end of $\S 2$ it follows that (3.5) implies the MacMahon Master Theorem. We prefer, however, to preserve the theorem in the form (3.5), because, as will be seen later, it yields a generalization.

3.4 Good's proof to Dyson's conjecture. In 1962, Dyson [2] made the following conjectures:

Conjecture B.

$$
\text { The constant term in }\left(\prod_{\substack{i \neq j \\ i \leqq i, j \leqq n}}\left(1-\frac{x_{i}}{x_{j}}\right)\right)^{a} \text { is }(n a) ! /(a !)^{n}
$$

and its generalization

Conjecture $\mathrm{C}$.

$$
\text { The constant term in } \prod_{\substack{i \neq j \\ 1 \leqq i, j \leqq n}}\left(1-\frac{x_{i}}{x_{j}}\right)^{a_{i}} \text { is } \frac{\left(a_{1}+\cdots+a_{n}\right) !}{a_{1} ! \cdots a_{n} !} \text {. }
$$

This was proved by Gunson [5], Wilson [9], and Good [4]. Good's proof is the proof that got us started in this business. Because of its importance, and also because of its elegance, we shall repeat it, in our notation. Set

$$
F\left(a_{1}, \cdots, a_{n} ; x_{1}, \cdots, x_{n}\right)=\prod_{\substack{i \neq j \\ 1 \leqq i, j \leqq n}}\left(1-\frac{x_{i}}{x_{j}}\right)^{a_{i}} .
$$

$F$ satisfies the $n$ partial difference equations,

$$
X_{i}^{-1} F=\left[\prod_{j \neq i}\left(1-\frac{x_{i}}{x_{j}}\right)\right]^{-1} F, \quad i=1, \cdots, n, \quad a_{i}>0 .
$$

Elimination of $x_{1}, \cdots, x_{n}$ yields

$$
\left(I-X_{1}^{-1}-\cdots-X_{n}^{-1}\right) F=0 \quad \text { in } a_{1}, \cdots, a_{n}>0 .
$$


This equation is independent of $x_{1}, \cdots, x_{n}$, and therefore (3.6) is also satisfied by $F_{0}\left(a_{1}, \cdots, a_{n}\right)$, the constant term of $F$. So

$$
\left(I-X_{1}^{-1}-\cdots-X_{n}^{-1}\right) F_{0}=0 .
$$

$G\left(a_{1}, \cdots, a_{n}\right)=\left(a_{1}+\cdots+a_{n}\right) ! /\left(a_{1} ! \cdots a_{n} !\right)$ is also a solution of $(3.6)$ and the boundary values match, by the inductive hypothesis. So it follows that $F_{0}\left(a_{1}, \cdots, a_{n}\right)=$ $\left(a_{1}+\cdots+a_{n}\right) ! /\left(a_{1} ! \cdots a_{n} !\right)$.

3.5 There are many generalizations to Dyson's conjecture B. In Dyson's conjecture $\mathrm{C}$ the factors $\left(1-\left(x_{i} / x_{1}\right)\right), \cdots,\left(1-\left(x_{i} / x_{n}\right)\right),(i=1, \cdots, n)$ were grouped together, but we can take any $n$ subsets of the $n(n-1)$ factors $\left(1-\left(x_{i} / x_{j}\right)\right)(i \neq j, 1 \leqq i$, $j \leqq n$ ) and group them together, forming a function of $a_{1}, \cdots, a_{n}, x_{1}, \cdots, x_{n}$. Then we can use elimination to find a partial difference equation independent of $x_{1}, \cdots, x_{n}$, satisfied by that function, and therefore also satisfied by the constant term (or any other coefficient, for that matter). Let us illustrate it by the following.

FACT. $F_{0}(a, b, c)=$ constant term in $F\left(x_{1}, x_{2}, x_{3}, a, b, c\right)$

$$
=\left[\left(1-\frac{x_{1}}{x_{2}}\right)\left(1-\frac{x_{2}}{x_{1}}\right)\right]^{a}\left[\left(1-\frac{x_{1}}{x_{3}}\right)\left(1-\frac{x_{3}}{x_{1}}\right)\right]^{b}\left[\left(1-\frac{x_{2}}{x_{3}}\right)\left(1-\frac{x_{3}}{x_{2}}\right)\right]^{c},
$$

is given by,

$$
G(a, b, c)=(2 a) !(2 b) !(2 c) !(a+b+c) ! /[a ! b ! c !(a+b) !(a+c) !(b+c) !] .
$$

Proof. $F$ satisfies the following linear partial difference equations,

$$
\begin{aligned}
& X_{1} F=\left(2-\frac{x_{1}}{x_{2}}-\frac{x_{2}}{x_{1}}\right) F \\
& X_{2} F=\left(2-\frac{x_{1}}{x_{3}}-\frac{x_{3}}{x_{1}}\right) F \\
& X_{3} F=\left(2-\frac{x_{2}}{x_{3}}-\frac{x_{3}}{x_{2}}\right) F
\end{aligned}
$$

Eliminating $x_{1}, x_{2}, x_{3}$ we get that $F$ satisfies the partial difference equation

$$
\left(X_{1}^{2}+X_{2}^{2}+X_{3}^{2}-2 X_{1} X_{2}-2 X_{1} X_{3}-2 X_{2} X_{3}+X_{1} X_{2} X_{3}\right) F=0 \text {. }
$$

Since this is a linear equation and is independent of $x_{1}, x_{2}, x_{3}$, it is also satisfied by $F_{0}(a, b, c)$. It is straightforward (albeit rather long) to check that this equation is also satisfied by $G(a, b, c)$. It is trivial to check that $F_{0}=G$ on the boundary of $Z_{+}^{3}$, $\{a=0\} \cup\{b=0\} \cup\{c=0\}$.

The special case $a=b=c$ yields conjecture $\mathrm{B}$ for $n=3$. The above proof was given for pedagogical reasons, because the Fact is equivalent to

$$
\sum(-1)^{k}\left(\begin{array}{c}
2 a \\
a+k
\end{array}\right)\left(\begin{array}{c}
2 b \\
b+k
\end{array}\right)\left(\begin{array}{c}
2 c \\
c+k
\end{array}\right)=\frac{(2 a) !(2 b) !(2 c) !(a+b+c) !}{a ! b ! c !(a+b) !(a+c) !(b+c) !}
$$

this is equivalent to the terminating form of Dixon's theorem, which in turn is equivalent to Dyson's conjecture C for $n=3$ (Andrews [1, p. 214]).

\section{A generalization of MacMahon's Master Theorem.}

4.1 In $\S 6$ we shall describe how to generalize the elimination procedure to systems of linear partial difference operators with variable coefficients. In this case the task is much harder, since the ring of linear partial difference operators is not commutative. 
However, in some cases of variable coefficients operators the elimination method generalizes right away. This happens when the operators to be eliminated are pairwise commutative. In particular, let us prove the following generalization of MacMahon's Master Theorem.

THEOREM. Let $\left(f_{i j}\left(m_{i}\right)\right), 0 \leqq i, j \leqq n$, be a matrix of discrete functions (where the $i$ th row has functions depending only on $m_{i}$ ), and for a discrete function $G$, let

$$
G^{(m)}=G(0) G(1) \cdots G(m-1) .
$$

Then $F_{0}\left(m_{1}, \cdots, m_{n}\right)=$ the constant term of

$$
F\left(m_{1}, \cdots, m_{n} ; x_{1}, \cdots, x_{n}\right)=\prod_{i=1}^{n}\left(\sum_{j=1}^{n} f_{i j} \frac{x_{j}}{x_{i}}\right)^{\left(m_{i}\right)}
$$

is given by

$$
G\left(m_{1}, \cdots, m_{n}\right)=\left[\operatorname{det}\left(\delta_{i j}-f_{i j} X_{i}^{-1}\right)\right]^{-1} \delta,
$$

where $\delta$ is the discrete Dirac delta function. $\operatorname{det}\left(\delta_{i j}-f_{i j} X_{i}^{-1}\right)$ is a linear partial difference operator with variable coefficients, and its inverse is calculated by (2.5).

Proof. The proof is along the same lines as the one given in subsection 3.3. $F=F\left(m_{1}, \cdots, m_{n} ; x_{1}, \cdots, x_{n}\right)$ satisfies

i.e.,

$$
X_{i} F=\left(\sum_{j=1}^{n} f_{i j}\left(m_{i}\right) \frac{x_{j}}{x_{i}}\right) F, \quad i=1, \cdots, n ;
$$

$$
\sum_{j=1}^{n} x_{j}\left[\delta_{i j} X_{i}-f_{i j}\left(m_{i}\right)\right] F=0, \quad i=1, \cdots, n .
$$

Gaussian elimination still works here because any two entries in the matrix $\left(\delta_{i j} X_{i}-f_{i j}\left(m_{i}\right)\right)$ which are in different rows, commute, since $X_{i}$ acts only on functions independent of $m_{i}$. Consequently $F\left(m_{i}, \cdots, m_{n} ; x_{i}, \cdots, x_{n}\right)$ satisfies

$$
\operatorname{det}\left(\delta_{i j} X_{i}-f_{i j}\left(m_{i}\right)\right) F=0 .
$$

This partial difference equation is independent of $x_{1}, \cdots, x_{n}$, and since it is linear it follows that it is also satisfied by $F_{0}\left(m_{1}, \cdots, m_{n}\right)$, the constant term of $F$.

So

$$
\operatorname{det}\left(\delta_{i j} X_{i}-f_{i j}\left(m_{i}\right)\right) F_{0} \equiv 0 \text {. }
$$

But we also have

$$
\operatorname{det}\left(\delta_{i j} X_{i}-f_{i j}\left(m_{i}\right)\right) G \equiv 0 .
$$

$F_{0}=G$ on the boundary of $Z_{+}^{n}$, that is on $\bigcup_{i=1}^{n}\left\{m_{i}=0\right\}$, by the inductive hypothesis, and thus the theorem follows: $F_{0} \equiv G$ throughout $Z_{+}^{n}$.

4.2 A $q$-analogue of MacMahon's Master Theorem. The above theorem answers, in particular, a question raised by Andrews [1, p. 213] about a $q$-analogue to MacMahon's Master Theorem. The $q$-analogue of $(a+b)^{m}$ is $(a+b)(a+q b)(a+$ $\left.q^{2} b\right) \cdots\left(a+q^{m-1} b\right)$; and naturally the $q$-analogue of $(a+b+c)^{m}$ would be $(a+b+$ c) $\left(a+q b+q^{2} c\right)\left(a+q^{2} b+q^{4} c\right) \cdots\left(a+q^{m-1} b+q^{2 m-2} c\right)$, and in general, a $q$-analogue of $\left(a_{1}+\cdots+a_{n}\right)^{m}$ would be

$$
\begin{aligned}
\left(a_{1}+\cdots+a_{n}\right)\left(a_{1}\right. & \left.+q a_{2}+\cdots+q^{n-1} a_{n}\right) \\
& \times\left(a_{1}+q^{2} a_{2}+\cdots+q^{2(n-1)} a_{n}\right) \cdots\left(a_{1}+q^{m-1} a_{2}+\cdots+q^{(m-1)(n-1)} a_{n}\right) .
\end{aligned}
$$


It is seen that a $q$-analogue of the Master Theorem is obtained by putting $f_{i j}\left(m_{i}\right)=$ $a_{i j} q^{m_{i}(j-1)}$, where the $a_{i j}$ 's are constants.

5. About the possibility of proving a $q$-analogue of Dyson's conjecture. In $[1, p$. 216], Andrews conjectured that the constant term of

$$
F=\prod_{\substack{i \neq j \\ 1 \leqq i, j \leqq n}}\left(\frac{\varepsilon_{i j} x_{i}}{x_{j}}\right)_{a_{i}}, \quad \varepsilon_{i j}= \begin{cases}1, & i<j, \\ q, & j>i,\end{cases}
$$

is $q_{a_{i}+\cdots+a_{n}} /\left(q_{a_{1}} \cdots q_{a_{n}}\right)$, where $(x)_{a}=(1-x)(1-q x) \cdots\left(1-q^{a-1} x\right)$. This is the $q-$ analogue of Dyson's conjecture C. Let us try to generalize Good's proof. We have the $n$ equations

$$
\begin{aligned}
& X_{1} F=\left(1-q^{a_{1}} \frac{x_{1}}{x_{2}}\right) \cdots\left(1-q^{a_{1}} \frac{x_{1}}{x_{n}}\right) F \\
& X_{2} F=\left(1-q^{a_{2}+1} \frac{x_{2}}{x_{1}}\right)\left(1-q^{a_{2}} \frac{x_{2}}{x_{3}}\right) \cdots\left(1-q^{a_{n}} \frac{x_{2}}{x_{n}}\right) F, \\
& \vdots \\
& X_{n} F=\left(1-q^{a_{n}+1} \frac{x_{n}}{x_{1}}\right) \cdots\left(1-q^{a_{n}+1} \frac{x_{n}}{x_{n-1}}\right) F .
\end{aligned}
$$

By finding the expressions for $X_{1}^{\alpha_{1}} \cdots X_{n}^{\alpha_{n}}$ for high enough $\alpha_{1}, \cdots, \alpha_{n}$, it is possible in principle (for a fixed $n$ ), to eliminate $x_{1}, \cdots, x_{n}$ from these equations, and get a linear partial difference operator $P\left(a_{1}, \cdots, a_{n} ; X_{1}, \cdots, X_{n}\right)$ such that $P F \equiv 0$. Then it would be possible to check that $P\left(q_{a_{1}+\cdots+a_{n}} /\left(q_{a_{1}} \cdots q_{a_{n}}\right)\right)=0$, and equate boundary values. Details will appear elsewhere.

However, this process is very complicated to do by hand (a symbolic computer will help here), and we were unable to find such an equation even for $n=3$.

\section{Elimination in the algebra of linear partial difference operators.}

6.1. Gaussian elimination in the ring of linear ordinary difference operators. Since the process of elimination in Van der Waerden [8] is based on Gaussian elimination in a commutative ring, we shall first describe how to modify Gaussian elimination to the noncommutative ring of partial difference operators. To begin with, let us consider the ring of linear ordinary difference operators.

Suppose we have the two operators

$$
\begin{aligned}
& Q_{1}=(a X+b) \lambda_{1}+P_{2}(m, X) \lambda_{2}+\cdots+P_{n}(m, X) \lambda_{n}, \\
& Q_{2}=\left(a^{\prime} X+b^{\prime}\right) \lambda_{1}+P_{2}^{\prime}(m, X) \lambda_{2}+\cdots+P_{n}^{\prime}(m, X) \lambda_{n},
\end{aligned}
$$

where $P_{2}, \cdots, P_{n}, P_{2}^{\prime}, \cdots, P_{n}^{\prime}$ are any linear difference operators and the coefficients of $\lambda_{1}$ are first order. If $a, b, a^{\prime}, b^{\prime}$ were constants we could have just multiplied the first equation by $\left(a^{\prime} X+b^{\prime}\right)$, the second by $(a X+b)$ and subtracted, thus getting rid of $\lambda_{1}$. But since $a, a^{\prime}, b, b^{\prime}$ are functions, $(a X+b)$ and $\left(a^{\prime} X+b^{\prime}\right)$ do not commute in general.

We first form

$$
\begin{aligned}
& b^{\prime} Q_{1}-b Q_{2}=\phi(m) X \lambda_{1}+\cdots, \\
& a^{\prime} Q_{1}-a Q_{2}=-\phi(m) \lambda_{1}+\cdots .
\end{aligned}
$$

Now we apply $X$ to (6.2b) and get

$$
X\left(a^{\prime} Q_{1}-a Q_{2}\right)=-[X \phi(m)] X \lambda_{1}+\cdots
$$


Since $\phi$ and $X \phi$ are functions, they commute, and $(X \phi)(6.2 \mathrm{a})+\phi\left(6.2 \mathrm{~b}^{\prime}\right)$ yields an operator independent of $\lambda_{1}$.

The above process can be described as reducing two equations in which the coefficients of $\lambda_{1}$ are first order difference operators, to two equations in $\left(X \lambda_{1}, \lambda_{2}, \cdots, \lambda_{n}\right)$ in which the coefficients of $X \lambda_{1}$ are zero order difference operators, i.e. functions.

The same method can be applied to the case in which the coefficients of $\lambda_{1}$ are of any given order $k$.

$$
\begin{aligned}
& Q_{1}=\left(a_{0} X^{k}+a_{1} X^{k-1}+\cdots+a_{k}\right) \lambda_{1}+\cdots, \\
& Q_{2}=\left(a_{0}^{\prime} X^{k}+a_{1}^{\prime} X^{k-1}+\cdots+a_{k}^{\prime}\right) \lambda_{1}+\cdots
\end{aligned}
$$

Now

$$
\begin{aligned}
& a_{0}^{\prime} Q_{1}-a_{0} Q_{2}=\left(b_{1} X^{k-1}+\cdots+b_{k}\right) \lambda_{1}+\cdots, \\
& a_{k}^{\prime} Q_{1}-a_{k} Q_{2}=\left(c_{0} X^{k-1}+\cdots+c_{k-1}\right) X \lambda_{1}+\cdots .
\end{aligned}
$$

Now applying $X$ to (6.4a) we get two operators, linear in $\left(X \lambda_{1}\right), \lambda_{2}, \cdots, \lambda_{n}$, in which the coefficients of $\left(X \lambda_{1}\right)$ are operators of order $k-1$. Continuing in this manner, we get rid of $\lambda_{1}$ altogether.

If we have $n$ operators, $Q_{1}, \cdots, Q_{n}$ linear in $\lambda_{1}, \cdots, \lambda_{n}$, we can use the above procedure to get $n-1$ operators linear in $\lambda_{2}, \cdots, \lambda_{n}$. Continuing in this manner, we end up with one operator linear in $\lambda_{n}$, and since it is possible to divide by $\lambda_{n}$, we end up with an operator $Q$ independent of $\lambda_{1}, \cdots, \lambda_{n}$. This operator is in the ideal generated by $\left\{Q_{1}, \cdots, Q_{n}\right\}$, and so $Q_{1} f=0, \cdots, Q_{n} f=0 \Rightarrow Q f=0$.

6.2 Gaussian elimination in the ring of linear partial difference operators. Let us consider a special case first,

$$
\begin{aligned}
& Q_{1}=[(a Y+b) X+(c Y+d)] \lambda_{1}+P_{2}(m, X, Y) \lambda_{2}+\cdots+P_{n}(m, X, Y) \lambda_{n}, \\
& Q_{2}=\left[\left(a^{\prime} Y+b^{\prime}\right) X+\left(c^{\prime} Y+d^{\prime}\right)\right] \lambda_{1}+P_{2}^{\prime}(m, X, Y) \lambda_{2}+\cdots+P_{n}(m, X, Y) \lambda_{n} .
\end{aligned}
$$

Let us write it as follows,

$$
\begin{aligned}
& Q_{1}=(a Y+b)\left(X \lambda_{1}\right)+(c Y+d) \lambda_{1}+\cdots, \\
& Q_{2}=\left(a^{\prime} Y+b\right)\left(X \lambda_{1}\right)+\left(c^{\prime} Y+d^{\prime}\right) \lambda_{1}+\cdots .
\end{aligned}
$$

Using the process of subsection 6.1 we get rid of $X \lambda_{1}$, to get an operator

$$
Q_{1}^{\prime}=P_{1}(Y) \lambda_{1}+\cdots \text {. }
$$

Once again using the above process, this time to get rid of $\lambda_{1}$, yields

$$
Q_{2}^{\prime}=P_{2}(Y)\left(X \lambda_{1}\right)+\cdots \cdot
$$

$X Q_{1}^{\prime}$ and $Q_{2}^{\prime}$ are two operators, linear in $\left(X \lambda_{1}\right), \lambda_{2}, \cdots, \lambda_{n}$, but with the advantage that the coefficients of $\left(X \lambda_{1}\right)$ are just ordinary difference operators. In this form it is possible to use the method in the previous subsection, to get rid of $\left(X \lambda_{1}\right)$ altogether.

In general, suppose that we know how to do Gaussian elimination for partial difference operators of dimension $K-1$; let us describe how to perform Gaussian elimination for partial difference operators of dimension $K$. Consider the two operators

$$
\begin{aligned}
& A=P_{1}\left(X_{1}, \cdots, X_{K}\right) \lambda_{1}+P_{2}\left(X_{1}, \cdots, X_{K}\right) \lambda_{2}+\cdots+P_{n}\left(X_{1}, \cdots, X_{K}\right) \lambda_{n} \\
& B=P_{1}^{\prime}\left(X_{1}, \cdots, X_{K}\right) \lambda_{1}+P_{2}^{\prime}\left(X_{1}, \cdots, X_{K}\right) \lambda_{2}+\cdots+P_{n}^{\prime}\left(X_{1}, \cdots, X_{K}\right) \lambda_{n}
\end{aligned}
$$


Let us write

$$
\begin{aligned}
& P_{1}\left(X_{1}, \cdots, X_{K}\right)=\sum_{i=0}^{L} Q_{i}\left(X_{2}, \cdots, X_{K}\right) X_{1}^{i}, \\
& P_{1}^{\prime}\left(X_{1}, \cdots, X_{K}\right)=\sum_{i=0}^{L} Q_{i}^{\prime}\left(X_{2}, \cdots, X_{K}\right) X_{1}^{i} .
\end{aligned}
$$

Substituting in (6.5) we get that $A, B$ are linearly dependent on $\lambda_{1}$, $\left(X_{1} \lambda_{1}\right), \cdots,\left(X_{1}^{L} \lambda_{1}\right), \lambda_{2}, \cdots, \lambda_{n}$. Using the algorithm for dimension $K-1$, we can get rid of $\lambda_{1}$, yielding

$$
C=C_{1}\left(X_{1} \lambda_{1}\right)+\cdots+C_{L}\left(X_{1}^{L} \lambda_{1}\right),
$$

and using it another time, we can get rid of $\left(X_{1}^{L} \lambda_{1}\right)$ :

$$
D=D_{1} \lambda_{1}+\cdots+D_{L-1}\left(X_{1}^{L-1} \lambda_{1}\right) \text {. }
$$

$C, X_{1} D$ are two linear operators in $\left(X_{1} \lambda_{1}\right), \cdots,\left(X_{1}^{L} \lambda_{1}\right)$. Continuing the process, we can dispose of $\left(X_{1} \lambda_{1}\right),\left(X_{1}^{2} \lambda_{1}\right), \cdots,\left(X_{1}^{L} \lambda_{1}\right)$ successively, and finally get rid of $\lambda_{1}$ altogether. This algorithm eventually yields an operator which is independent of $\lambda_{1}, \cdots, \lambda_{n}$.

6.3 General elimination. Let us recall that if $P_{1}, P_{2} \in R\left[x_{1}, \cdots, x_{n}\right]$, where $R$ is a commutative ring, we get an element $Q \in\left\{P_{1}, P_{2}\right\}$, which is in $R\left[x_{2}, \cdots, x_{n}\right]$, by expressing $P_{1}, P_{2}$ as polynomials in $x_{1}$, with coefficients in $R\left[x_{2}, \cdots, x_{n}\right]$, and by forming $x_{1}^{l} P_{1}, x_{1}^{l} P_{2}$ for sufficiently large $l$, thus getting linear equations in the powers of $x_{1}$, with coefficients in $R\left[x_{2}, \cdots, x_{n}\right]$; then we use Gaussian elimination. The same method can be used in $\mathscr{P}_{n}\left[x_{1}, \cdots, x_{n}\right]$, where $\mathscr{P}_{n}$ is the ring of partial difference operators on $Z^{n}$. This is so because we know how to perform Gaussian elimination in that ring.

In general, if we have $N+1$ operators $P_{1}\left(x_{1}, \cdots, x_{N}\right), \cdots, P_{N+1}\left(x_{1}, \cdots, x_{N}\right)$, where the dependence on $x_{1}, \cdots, x_{N}$ is polynomial, it is possible to get an operator which belongs to the ideal $\left\{P_{1}, \cdots, P_{N+1}\right\}$, and which is independent of $x_{1}, \cdots, x_{N}$. The present algorithm is a generalization to the ring of linear partial difference operators, of the process described in subsection 3.2 for the ring of partial difference operators with constant coefficients.

6.4 Overdetermined systems of linear partial difference operators. In subsection 3.1 we saw that two linear partial difference operators with constant coefficients usually give rise to an operator of lower dimension. The same is true for general linear partial difference operators. Let $P, Q$ be two such operators on $Z^{n}$, and write them as follows,

$$
\begin{aligned}
& P=\sum P_{i}\left(X_{2}, \cdots, X_{n}\right) X_{1}^{i}, \\
& Q=\sum Q_{i}\left(X_{2}, \cdots, X_{n}\right) X_{1}^{i} .
\end{aligned}
$$

By considering $P, X_{1} P, \cdots, X_{1}^{L} P, Q, X_{1} Q, \cdots, X_{1}^{M} Q$ for sufficiently large $L, M$ we get linear dependence on the powers of $X_{1}$ and using the process of Gaussian elimination described in subsections $6.1,6.2$, we obtain an operator involving only $X_{2}, \cdots, X_{n}$, which is in the ideal $\{P, Q\}$. In general if we have $n$ operators $P_{1}\left(X_{1}, \cdots, X_{n}\right), \cdots, P_{n}\left(X_{1}, \cdots, X_{n}\right)$ we should get $n$ "ordinary" operators $Q_{1}\left(X_{1}\right), \cdots, Q_{n}\left(X_{n}\right)$. If this is the case, the ideal $\left\{P_{1}, \cdots, P_{n}\right\}$ is called "complete intersection". If this is not the case, then the algorithm will tell us so by breaking down. 
Example. Find 2 ordinary difference operators satisfied by every solution of the system

$$
\begin{aligned}
& m f(m+1, n+1)+n f(m+1, n)+2 m f(m, n+1)-m n f(m, n)=0, \\
& f(m+1, n+1)+(n+3) f(m+1, n)+m f(m, n+1)-3 m f(m, n)=0
\end{aligned}
$$

$m, n \geqq 0$.

In our notation

$$
\begin{aligned}
& P_{1}(X, Y) f=(m X Y+n X+2 m Y-m n I) f=0, \\
& P_{2}(X, Y) f=(X Y+(n+3) X+m Y-3 m I) f=0 .
\end{aligned}
$$

We have to eliminate $P_{1}, P_{2}$. Writing

$$
\begin{aligned}
& P_{1}=m X Y+2 m Y+(n X-m n I), \\
& P_{2}=X Y+m Y+(n+3) X-3 m I,
\end{aligned}
$$

yields

$$
\begin{aligned}
& Q_{1}=P_{1}-m P_{2}=\left(2 m-m^{2}\right) Y+\left(n X-m n I-m(n+3) X+3 m^{2} I\right), \\
& Q_{2}=P_{1}-2 P_{2}=(m-2) X Y+(-(n+6) X+6 m-m n) .
\end{aligned}
$$

Simplifying, we get

$$
\begin{aligned}
& Q_{1}=m(2-m) Y+(n-m n-3 m) X+m(3 m-n) I, \\
& Q_{2}=(m-2) X Y+(-(n+6) X+(6-n) m I) .
\end{aligned}
$$

So,

$$
\begin{aligned}
X Q_{1} & =\left(1-m^{2}\right) X Y-(m n+3 m+3) X^{2}+(m+1)(3 m-n+3) X, \\
Q_{2} & =(m-2) X Y+(-(n+6) X+(6-n) m) .
\end{aligned}
$$

$(m-2) X Q_{1}-\left(1-m^{2}\right) Q_{2}$ will be an ordinary difference operator in the $m$-direction, i.e., an operator in $X$. Similarly, it is possible to find an ordinary difference operator in the $n$-direction.

Remark. In Even and Gillis [3] the authors mention that they were unable to find a combinatorial proof of the ordinary difference equation (34) there, which is satisfied by $P_{r s t}$. However they exhibit 3 partial difference equations satisfied by $P_{r s t}$, namely (9), (32), and one obtained from (32) by replacing $r$ by $s$. Thus our (completely elementary) algorithm should yield their ordinary difference equation (34).

\section{REFERENCES}

[1] George E. Andrews, Problems and prospects for basic hypergeometric functions, in Theory and Applications of Special Functions, Richard A. Askey, ed., Academic Press, New York, 1975, pp. 191-224.

[2] F. J. Dyson, Statistical theory of the energy levels of complex systems I, J. Math. Phys., 3 (1962), pp. 140-156.

[3] S. Even And J. Gillis, Derangements and Laguerre polynomials, Math. Proc. Cambridge Philos. Soc., 79 (1976), pp. 135-143.

[4] I. J. GooD, Short proof of a conjecture of Dyson, J. Math. Phys., 11 (1970), p. 1884.

[5] J. Gunson, Proof of a conjecture by Dyson in the statistical theory of energy levels, J. Math. Phys., 3 (1962), pp. 752-753.

[6] LARS HöRMANDER, Linear Partial Differential Operators, Springer, Berlin, 1963. 
[7] P. A. MacMahon, Combinatory Analysis, Vol. 1, Cambridge University Press, Cambridge, 1915, (reprinted by Chelsea, New York, 1960).

[8] B. L. VAN Der WAerden, Modern Algebra, Vols. 1, 2, F. Blum, transl., Frederick Ungar Publishing Co., New York, 1953.

[9] K. Wilson, Proof of a conjecture by Dyson, J. Math. Phys., 3 (1962), pp. 1040-1043. 